\title{
Discrete breathers above phonon spectrum
}

\author{
V. Hizhnyakov ${ }^{1}$, M. Haas ${ }^{1}$, M. Klopov², A. Shelkan ${ }^{1, \dagger}$ \\ ${ }^{\dagger}$ shell@ut.ee \\ ${ }^{1}$ Institute of Physics, University of Tartu, Ravila 14c, 50411 Tartu, Estonia \\ ${ }^{2}$ Department of Physics, Faculty of Science, Tallinn University of Technology, Ehitajate 5, 19086 Tallinn, Estonia
}

\begin{abstract}
It is shown that in some metals $(\mathrm{Ni}, \mathrm{Nb}, \mathrm{Fe}, \mathrm{Cu})$ may exist discrete breathers with frequencies above the top of the phonon spectrum. These excitations are mobile: they may propagate along the crystallographic directions transferring energy of $\gtrsim 1 \mathrm{eV}$ over large distances. The discrete breathers with the frequencies above the top of the phonon bands may also exist in covalent crystals (diamond, Si and Ge). It is also found that in monatomic chains and planes (e.g. in graphene), the transverse discrete breathers may be excited above the spectrum of corresponding phonons. Although these vibrations are in resonance with longitudinal (chain) or in-plane (graphene) phonons the lifetime of them may be very long.
\end{abstract}

Keywords: nonlinear dynamics; anharmonic crystal lattice; discrete breathers.

\section{Introduction}

It is already a well known fact that in crystal lattices may exist long living anharmonic modes of rather high energy $\gtrsim 1 \mathrm{eV}$. These excitations are called as discrete breathers (DBs), intrinsic localized modes, vibrational solitons, or quodons $[2,4,7,11,13,14,16,17,29,31,32,34,42,43,46$, $47,48,51,52]$. In numerical studies of DBs different twobody potential models (Morse, Lennard-Jones, Born-MayerCoulomb and other potentials) have been used. All these potentials show strong softening at increasing vibrational amplitudes. Therefore the frequencies of DBs, found in these studies, drop down from the optical band(s) into the phonon gaps, if such gaps exist in the spectrum (see, e.g. Refs. [26, $28,30])$.

However the simple pair potentials approach should not work for all systems. E.g. in metals the interaction of ions is strongly affected by conducting electrons which cause the screening of it at intermediate and large distances. As a result of the screening the odd anharmonic terms in pair potentials may be essentially reduced that opens a possibility to split DBs up from the phonon spectrum [15]. The screening is a cooperative phenomenon which may be taken into account e.g. by applying the embedded atom model (EAM). Using this model, it was found that in some metals (Ni, Nb [15], Fe [20], $\mathrm{Cu}[19]$ ) indeed may exist the DBs with the frequencies above the phonon spectrum. These DBs can be both, not mobile and mobile.

In covalent crystals the interactions between atoms (ions) strongly depend on direction. It appeared that this dependence may lead to the reduction of the effect of the odd anharmonicities and to the hardening of anharmonic modes with increasing their amplitude. As a result in these crystals the DBs with the frequencies above the top of the phonon spectrum may exist; see e.g. Ref. [55] where this type DBs were found in Si and Ref. [19] where these type DBs were found in diamond using Tersoff-type potentials $[53,54]$.
In stretched atomic chains and planes (e.g. in graphene), due the lack of atoms in transverse direction the odd anarmonicities for the shifts are absent. Therefore for these systems there may exist localized anharmonic transverse modes with the frequencies above the spectrum of corresponding phonons [21]. Although these frequencies are in resonance with the longitudinal (chain) or in-plane (graphene) phonons, the modes can decay only due to weak anharmonic processes and therefore can have very long life time.

In this study we discuss the properties of DBs with the frequencies above the phonon spectrum (metals, semiconductors). We will show that in crystals with linear chains formed by nearest neighboring atoms such DBs can move along the crystallographic directions corresponding to the chains. Thereat they can transfer a large (as compared with a phonon quantum) amount of vibrational energy over long distances. We will also discuss the properties of transverse DBs in monatomic chains and planes (in graphene) split up from the top of the transverse phonoins. We will show that, although these modes are in resonance with longitudinal (chain) or in-plane (graphene) phonons the lifetime of them may be very long. Depending on initial conditions, the modes may stay or move.

\section{Discrete breathers above the phonon spectrum}

First we consider the anharmonic monatomic chain and discuss the vibrations of its atoms in longitudinal $(x)$ directions. We suppose that the potential energy of the chain is given by the sum of pair potentials $V\left(R_{n, n^{\prime}}\right)$, where $R_{n, n^{\prime}}$ is the distance between the atoms $n$ and $n$ '. The latter potentials can be expanded into the series of atomic displacements. In a linear chain with the nearest neighbor interactions $R_{n, n+1}=a+x$, where $a$ is the atom spacing (the length of the 
bond) in the equilibrium configuration, $x$ is the difference of the distance of the neighbouring atoms from its equilibrium value. In harmonic approximation with nearest neighbor interactions $\mathrm{V}=K_{2} x^{2} / 2$, and the vibrational frequencies of the longitudinal phonons equal [38]

$$
\omega_{k}=\sqrt{K_{2}(1-\cos k) / 2}
$$

( $k$ is the wave number of phonons). In Ref. [31] Kosevich and Kovalev considered longitudinal vibrations of the chain taking into account the cubic and quartic anharhonic terms $K_{3} x^{3} / 3$ and $K_{4} x^{4} / 4$ in the pair potential. They have shown that in such a chain there exist a solution of vibrational dynamics which describes the localized modes (called later as DBs) with the frequencies above the top of the phonon spectrum if

$$
\kappa=3 K_{2} K_{4} / 4 K_{3}^{2}>1 \text {. }
$$

In Ref. [15] it was shown that in this case the effective elastic spring of a bond in the chain equals $K_{2}+\delta K_{2}$, where

$$
\delta K_{2}=2 K_{3} \bar{\xi}+3 K_{4} \bar{A}^{2} / 4
$$

is the renormalization of the spring by the $\mathrm{DB}, \bar{A}$ is the vibrational amplitude of the bond and $\bar{\xi}=-\left(K_{3} / 2 K_{2}\right) \bar{A}^{2}$ is the change of the static length of the bond. Usually $K_{3}$ is negative and $\bar{\xi}$ is positive, i.e. a bond expands due to its vibration. The renormalization of $K_{2}$ is positive if the KosevichKovalev condition (2) is fulfilled. Thus, to split a DB upward from the top of the phonon spectrum, the renormalization $\delta K_{2}$ must be positive, i.e. the $\mathrm{DB}$ has to harden the bonds. It takes place if the quartic anharmonicity prevails over the effects of the cubic anharmonicity according to the condition (2). However for usual pair potentials (Morse, LennardJones, Born-Mayer-Coulomb), the opposite case takes place: $\kappa$ does not exceed even $1 / 2$. Therefore for such (usually called as "realistic") pair potentials one cannot expect to obtain DBs in chains with the frequencies above the phonon spectrum.

In $3 \mathrm{D}$ lattices, in comparison with the monoatomic chains the situation is changed due to the increase of the number of the actual bonds of an atom, the bonds with the more numerous distant neighbors inclusive. The result is the increasing stiffness of the lattice which in its turn leads to the reduced changes $\bar{\xi}$ of the static lengths and to the decreased stemming from these changes the negative term in $\delta K_{2}$. This circumstance may favor the existance of DBs with the frequencies above the phonon spectrum in 3D case.

The aforementioned arguments are essential for metals where such DBs indeed can exist. Two types of these excitations may be distinguished: 1) DBs appearing already at small amplitudes and low energy $E<0.5 \mathrm{eV}$, and 2) DBs existing at rather large amplitudes and high energy only. DBs with large amplitudes have noticeable non-harmonic character.

The first type of DBs, existing in monatomic fcc and bcc lattices, characterized by the vibrations along the chains of the nearest neighbor atoms renormalize the elastic force constants of the main vibrating bonds as follows: $\delta K_{2}=2 K_{3} \bar{\xi}+3 K_{4} \bar{A}^{2} / 4$. Here $\bar{\xi}=-\left(K_{3} / 2 \tilde{K}_{2}\right) \bar{A}^{2}$ is the expansion of the main vibrating bonds, $\tilde{K}_{2}=M v^{2} / r_{0}^{2}>K_{2}$ is the mean elastic force constant in the bulk, $v$ is the longitudinal velocity of sound, $r_{0}$ is the equilibrium nearest-neighbour distance [15] (note that in the periphery of a DB the bonds are contracted). Consequently, analogously to a $1 \mathrm{D}$ atomic chain, cubic anharmonicity results in the local expansion of the lattice. This expansion also gives a negative contribution to the elastic springs, although somewhat smaller than in the chain. To split a DB frequency from the top of the phonon spectrum upward, the effect of cubic anharmonicity should be less than that of quartic anharmonicity. Such a situation takes place if

$$
\tilde{\kappa}=3 \tilde{K}_{2} K_{4} / 4 K_{3}^{2}>1 \text {. }
$$

Let us notice that in a $3 \mathrm{D}$ lattice the effective renormalized elastic spring $K_{2}$ is stronger than $K_{2}$ in the corresponding linear chain and condition (4) may be easier fulfilled in comparison with the condition (2). However, as a rule $K_{2}$ exceeds $K_{2}$ only a little (roughly $10 \div 20 \%$ ) and the increase of the ordinary value of $\kappa \lesssim 0.5$ is not sufficient. The required increase may be possible only if the interaction between atoms is essentially different from that described by usual pair potentials. Possible candidates here are metals: due to the presence of free electrons and Friedel oscillations of the pair potentials caused by the screening of the interatomic interactions by these electrons cubic anharmonicity for small displacements of atoms from their equilibrium position. In Ref. [15] it was shown that in some metals, e.g. in metallic $\mathrm{Ni}$ and $\mathrm{Nb}$ the criterion (4) is indeed fulfilled for small displacements and the DBs with the frequency above the phonon spectrum do exist.

However it appeared that in some metals (e.g. in iron and copper) may exist DBs with large vibrational amplitudes and energy altough the criterion (4) is unfulfilled ( $\widetilde{\kappa}$ is remarkably less than unity) and DBs with small amplitude and energy cannot exist. The reason of that is as follows. As we have mentioned above, the factor preventing the existence of DBs with the frequencies above the phonon spectrum is the local expansion of the main vibrating bonds. In 3D lattices this expansion is localized, which means that it is practically stopped at the very nearest periphery of the DB. The required additional compressing forces are caused by the interactions with the atoms, positioned outside the main vibrating chain, and have superlinear dependence on the expansion. Thus, a possibility can arise to appear well-localized DBs with rather large vibrational amplitudes. The static changes of the lengths of the interatomic bonds in the actual central region of such a DB are not proportional to $\bar{A}^{2}$, the basic assumption of the criterion (4). The contribution of the higher harmonics may also become significant in such DBs.

Besides it appeares that in covalent crystals with diamond structure (diamond, $\mathrm{Si}, \mathrm{Ge}$ ) may also exist DBs with the frequency above the top of the phonon spectrum although the cubic anharmonicity of every bond in these compounds is remarkable $\left(\left|K_{3}\right|>\sqrt{K_{2} K_{4}}\right)$. Every atom in these structures is connected by strong covalent bonds with four atoms positioned at the vertexes of a tetrahedron. An essential property of these bonds is their strong orientation dependence resulting in strong resistance of the tetrahedrons against the distortion of their shape. Therefore the interactions with the atoms positioned out of the main vibrating bond direction reduce the local expansion which in its turn hinders the softening of the main bond. This makes possible the existence of DBs with the frequency above the top of the phonon spectrum in these structures. Note that the same structural peculiarity is the cause of the extremely small thermal expansion of these systems. 


\subsection{Numerical modelling of standing DBs in metals}

In our calculations of DBs in metals we use the embedded atom model (EAM) $[9,10]$, which takes the electron density into account. According to this model the potential energy of a crystal can be presented in the form

$$
E=\frac{1}{2} \sum_{n n^{\prime}} V\left(r_{n n^{\prime}}\right)+\sum_{n} F\left(\rho_{n}\right) .
$$

Here $V\left(r_{n n^{\prime}}\right)$ is a pair potential as a function of the distance $r_{n n^{\prime}}$, between atoms $n$ and $n$ ', the volume dependent functions $F$ represent the "embedding energies" via the "host density" functions $\rho_{n}=\Sigma_{n \neq n} \rho\left(r_{n n^{\prime}}\right)$ induced at site $n$ by all other atoms in the system. The "host density" is assumed to be composed of contributions of single host atoms ("atomic density" functions $\left.\rho\left(r_{n n^{\prime}}\right)\right)$. The division of $E$ into two terms can be done in such a way that the contribution of the second term will be small for configurations close to the equilibrium. Such division is appropriate for consideration of anharmonic forces in DBs: due to the short-range origin of these forces, their corrections induced by the second term in Eq. (5) are small. In contrast, the harmonic forces, as a rule more remarkably affected by extended interactions, may be influenced by the embedding energy more significantly. According to (5) one can reasonably calculate the force constants $\widetilde{K}_{2}, K_{3}$ and $K_{4}$ and use criterion (4) to estimate the possibility of the first type DBs in the system.

An example showing the feasibility of the criterion (4) is metallic nickel. The EAM potential of this metal is well known with rather high accuracy. The values of the anharmonic springs are $K_{2}=2.32 \mathrm{eV} / \AA^{2}, K_{3}=-11 \mathrm{eV} / \AA^{3}$ and $K_{4}=73 \mathrm{eV} / \AA^{4}$ [15]. This gives $\kappa=1.05$. This is much larger than one gets for common pair potentials in ionic crystals. The reason for that is small value of odd anharmonicity parameter $K_{3}$ which is a consequence of presence of conducting electrons in Ni. The longitudinal sound velocity in $\mathrm{Ni}$ at room temperature equals $v=5266 \mathrm{~m} / \mathrm{sec}$. This gives $\widetilde{K}_{2}=2.75 \mathrm{eV} / \AA^{2}$ and $\widetilde{\kappa}=1.24$. Hence, in $\mathrm{Ni}$ the condition $\widetilde{\kappa}>1$ is fulfilled. Taking into account that the approximation of the EAM potential by forth power polynomial works reasonably well for vibrations with amplitudes $<0.25 \AA$ one can expect that low energy DB can exist in this metal. MD simulations confirm this conclusion [15]. Another metal when DBs of small amplitude and with the frequencies above the top of the phonon spectrum can exist is metallic niobium [15]. In this metal also $\widetilde{\kappa}>1$ although it value is very close to the limit $\widetilde{\kappa}=1$.

In many other metals the criterion (4) is not fulfilled. However, as it has been mentioned above our MD simulations show that at least in some of such metals DBs may still exist; their properties well fit to the second type DBs described in the previous section. Good examples of such DBs have been found in copper $(\mathrm{Cu}, \mathrm{fcc}$ lattice). In this metal, the forth degree polynomial approximation of the first term of EAM potential proposed in $[41,56]$ gives for the force constants the values: $K_{2}=2.1 \mathrm{eV} / \AA^{2}, K_{3}=-5.8 \mathrm{eV} / \AA^{3}, K_{4}=6.9 \mathrm{eV} / \AA^{4}$. This gives $\kappa=0.3$, i.e. $\kappa$ is much less than unity. The corrected mean elastic force constant equals to $\widetilde{K}_{2}=2.32$ and the corresponding parameter $\widetilde{\kappa}=0.32$ is also much less than unity. Nevertheless, according to our simulations even DBs with the energy between $4.05 \mathrm{eV}$ and $6 \mathrm{eV}$ can exist in 3D Cu lattice.
Examples of such DBs are given on Figures 1 and 2. The data about the aforementioned DBs are collected in Table 1. Here the DB energies, frequencies, vibrational amplitudes (with initial phases) and the corresponding static shifts of a central atom and its five neighbors in the main atomic chain of DBs positioned along (110) axis (atoms $(n, n, 0), n=0,1,2,3,4,5)$ are presented. The displacements $u_{n}$ in the central chain of even DBs satisfy the symmetry conditions $u_{-n-1}=-u_{n}$. The atoms are vibrating in (110) direction. Note that in the case of the DB with the energy $E=4.05 \mathrm{eV}$, the frequency $v_{l}=8 \mathrm{THz}$ exceeds the top phonon frequency $v_{M}=7.9 \mathrm{THz}$ very slightly.

From these data one can clearly see the existence of the compression effect in the main atomic chain. Such compression reduces the expansion of the chain and compensates the effect of the elongation of the central interatomic bonds caused by the odd anharmonicity of the atomic forces. As it has been mentioned in the previous section, the result is the higher local stiffness of the lattice and the possibility to appear DBs with frequencies above the phonon spectrum. Consequently the properties of the $\mathrm{DB}$ in $\mathrm{Cu}$ indeed agree with the described above properties of the second type DBs.

Another example of the second type DB appears in the bcc lattice of iron. In EAM proposed for the bcc iron (Refs. $[8,56])$, the pair potential $V(r)$ near the equilibrium may be approximated by a polynomial of the forth degree determined via the harmonic and anharmonic force constants $K_{2}=2.81 \mathrm{eV} / \AA^{2}, K_{3}=-12 \mathrm{eV} / \AA^{3}, K_{4}=45.2 \mathrm{eV} / \AA^{4}$. The corrected effective harmonic force constant in this metal equals to $\widetilde{K}_{2}=3.33 \mathrm{eV} / \AA^{2}$. The corresponding parameters $\kappa=0.66$ and $\widetilde{\kappa}=0.78$ are less than unity although much closer to this value than in $\mathrm{Cu}$. By analogy the existence of DBs above the phonon spectrum may be also expected in this metal although with more modest energies than in $\mathrm{Cu}$. Our numerical simulations confirm this expectation as even DBs with energy between $0.5 \mathrm{eV}$ and $3.5 \mathrm{eV}$ were indeed generated in our computations (see Figure 3). The frequencies of the small energy DBs are close to the maximum phonon frequency (see Figure 4 and Table 2).

To verify the $3 \mathrm{D}$ origin of the $\mathrm{DBs}$ in $\mathrm{Cu}$ and $\mathrm{Fe}$ we performed also simulations of nonlinear dynamics in $1 \mathrm{D} \mathrm{Cu}$ and Fe lattices with the same pair atomic interactions as in the $3 \mathrm{D}$ case. Indeed the calculations prove that in these $1 \mathrm{D}$ lattices DBs with neither small nor large amplitudes can be generated.

\section{Standing DBs in Ge and diamond}

DBs with the frequencies located above the phonon spectrum may also exist in covalent crystals with diamond structure. Such DBs appeared in the numerical simulations of Voulgarakis et al. [55] in Si whereby the atomic potential, proposed by Tersoff $[53,54]$, was applied. We have performed similar calculations for Ge which results are given in Figure 5.

The lattice structure of these crystals is presented by two fcc lattices, shifted with respect to each other by the vector $a_{0}(1 / 4,1 / 4,1 / 4)\left(a_{0}\right.$ is fcc lattice constant) whereby the nearest neighbors of every atom are positioned in the vertexes of a tetrahedron and no atomic chain formed by nearest neighbor atoms can exist. In these systems the atomic forces 


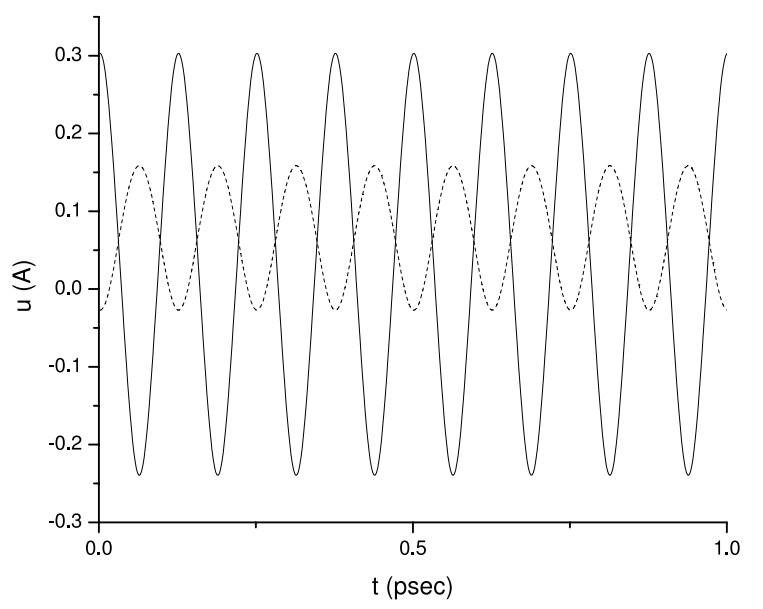

Fig. 1. $\mathrm{DB}$ in $\mathrm{Cu}$ : time dependence of the vibration of the central atom $(n=0)$ and the third side atom $(n=3)$ along (110) axis with the frequency $8 \mathrm{THz}$.

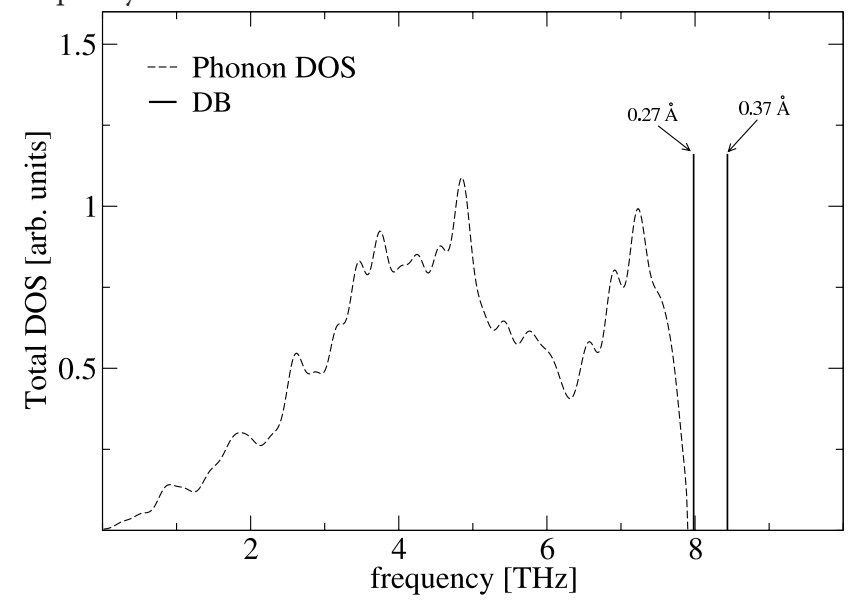

Fig. 2. Spectra of vibrations of $\mathrm{Cu}$ atoms in metallic $\mathrm{Cu}$ for two DBs with different frequencies and amplitudes in $(1,1,0)$ direction. The vibrational amplitudes of central atoms of DBs indicated by arrows.

Table 1. Even DBs in Cu. Energy, frequency and shape (vibrational amplitudes and static shifts of atoms in (110) central chain of DB, both in $\AA$ ).

\begin{tabular}{|c|c|c|c|c|c|c|c|}
\hline $\begin{array}{c}E \\
(\mathrm{eV})\end{array}$ & $\begin{array}{c}v \\
(\mathrm{THz})\end{array}$ & $\begin{array}{c}A_{0} \\
\xi_{0}\end{array}$ & $\begin{array}{c}A_{1} \\
\xi_{1}\end{array}$ & $\begin{array}{c}A_{2} \\
\xi_{2}\end{array}$ & $\begin{array}{c}A_{3} \\
\xi_{3}\end{array}$ & $\begin{array}{c}A_{4} \\
\xi_{4}\end{array}$ & $\begin{array}{c}A_{5} \\
\xi_{5}\end{array}$ \\
\hline \multirow{3}{*}{4.05} & \multirow{2}{*}{8.0} & 0.271 & -0.229 & 0.161 & -0.093 & 0.045 & -0.019 \\
& & 0.032 & 0.078 & 0.087 & 0.066 & 0.040 & 0.022 \\
\hline \multirow{3}{*}{6.0} & \multirow{2}{*}{8.4} & 0.373 & -0.314 & 0.218 & -0.120 & 0.049 & -0.016 \\
& & 0.061 & 0.154 & 0.175 & 0.135 & 0.080 & 0.043 \\
\hline
\end{tabular}

are essentially determined by the covalent chemical bonds characterized by strong dependence on direction. Due to the contribution of many-body forces, the interactions with the next to nearest and even next to next to nearest atoms may be significant. In these crystals the simple condition (4) is always violated. Because the basic assumptions of this condition (a quasi-chain character of the DB and the atomic interaction described by a simple pair potential determined via some force constants) are not justified here, it can be supposed that the resistance of the vibrating bonds against their expansion can exceed the softening of the bonds caused by the odd anharmonicity of them. Indeed, in our molecular

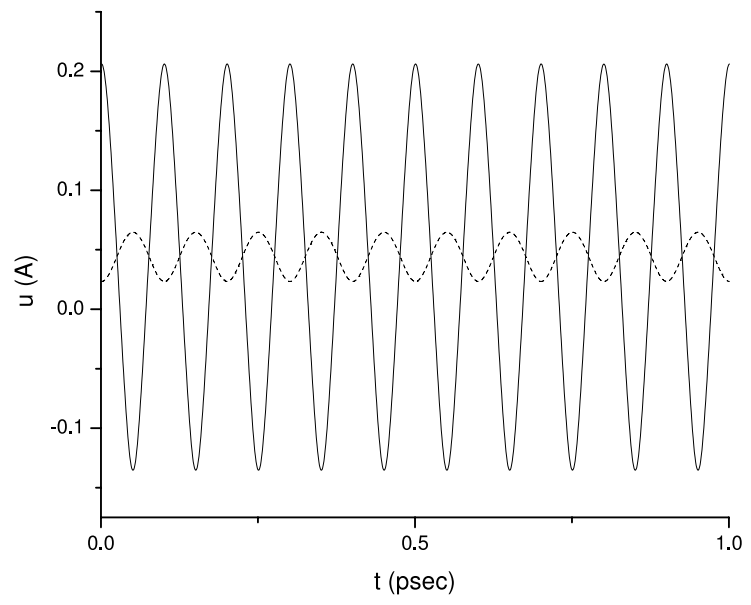

Fig. 3. DB in Fe: time dependence of the vibration of the central atom $(n=0)$ and the third side atom $(n=3)$ along (111) axis with the frequency $10 \mathrm{THz}$.

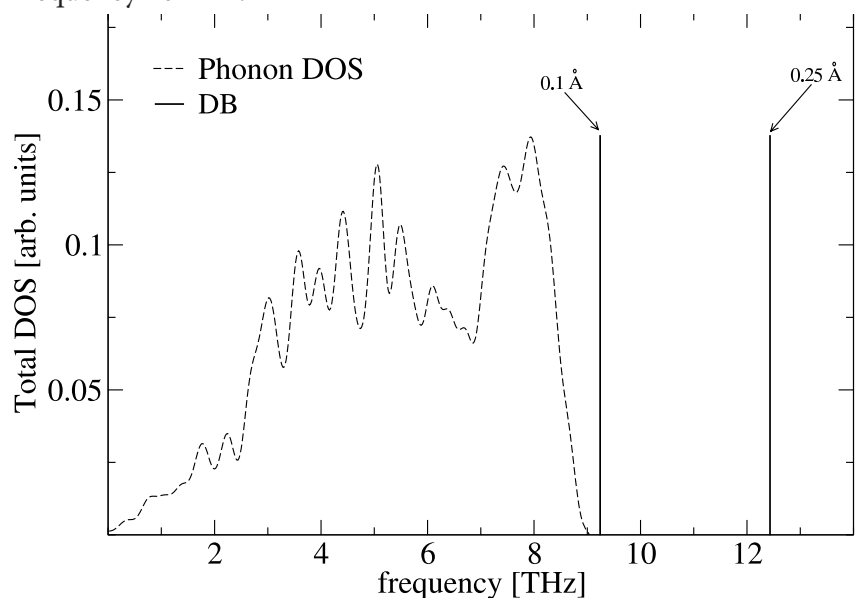

Fig. 4. Spectra of vibrations of Fe atoms in metallic Fe for two DBs with different frequencies and amplitudes in $(1,1,1)$ direction. The vibrational amplitudes of central atoms of DBs indicated by arrows.

Table 2. Even DBs in Fe. Energy, frequency and shape (vibrational amplitudes and static shifts of atoms in (111) central chain of DB, both in $\AA$ ).

\begin{tabular}{|c|c|c|c|c|c|c|c|}
\hline $\begin{array}{c}E \\
(\mathrm{eV})\end{array}$ & $\begin{array}{c}v \\
(\mathrm{THz})\end{array}$ & $\begin{array}{c}A_{0} \\
\xi_{0}\end{array}$ & $\begin{array}{c}A_{1} \\
\xi_{1}\end{array}$ & $\begin{array}{c}A_{2} \\
\xi_{2}\end{array}$ & $\begin{array}{c}A_{3} \\
\xi_{3}\end{array}$ & $\begin{array}{c}A_{4} \\
\xi_{4}\end{array}$ & $\begin{array}{c}A_{5} \\
\xi_{5}\end{array}$ \\
\hline & & & & & & & \\
0.5 & 9.3 & 0.099 & -0.075 & 0.045 & -0.022 & 0.010 & -0.004 \\
& & 0.011 & 0.025 & 0.024 & 0.016 & 0.009 & 0.005 \\
\hline & & & & & & & \\
3.5 & \multirow{2}{*}{12.4} & 0.249 & -0.202 & 0.125 & -0.056 & 0.016 & -0.003 \\
& & 0.070 & 0.179 & 0.208 & 0.165 & 0.103 & 0.059 \\
\hline
\end{tabular}

dynamic simulations, a well-located DB with the central bond between two nearest neighbor atoms along a (111) axis, arises in a Ge crystal. The frequency of $\mathrm{DB} \approx 10.07 \mathrm{THz}$ exceeds significantly the upper limit of the phonon spectrum $\sim 9.2$ THz. Thereby the interval of the large vibrational amplitudes of the central bond $(\sim 0.42 \AA)$, inducing DBs, is very limited even in comparison with the situation in $\mathrm{Si}$ [55] and the band of the DB frequencies is extremely narrow producing a large gap $(\sim 0.8 \mathrm{THz})$ with the phonon spectrum. The main reason of such DB is the strong stiffening of the central bond in its extreme, most elongated position due to the fast switching-off of the interatomic forces. The simulations have been carried 
out in a cluster in the form of a hexagonal prism elongated along (111) axis and containing 93500 atoms.

Analogously to the simulations of Ref. [55], our attempts to simulate $\mathrm{DB}$ in diamond using the Tersoff potential, failed. Even the positive results in Ge are connected with large amplitudes of DBs, i.e. with such interatomic distances where the aforementioned potential may not describe the interatomic forces adequately. Therefore we repeated the MD simulation in diamond using a more advanced LCBOP potential proposed recently in [33], which takes into account the bond orientation and the switching-off effects more accurately. Using this potential we have found rather good DBs (see Figure 6).

The MD simulations of $\mathrm{DBs}$ were performed in a rather big cluster $30 \times 30 \times 30$ ( 216000 carbon atoms). The total phonon density of states (DOS) was calculated for $100 \mathrm{~K}$ as a result of $6500000 \mathrm{MD}$ steps (time step $=0.002 \mathrm{psec}$ ) using periodical boundary conditions. To excite DB in diamond lattice the two nearest carbon atoms were shifted in the opposite phase along (111) direction. The DBs with frequencies above the phonon spectrum were generated at relatively small shifts already.

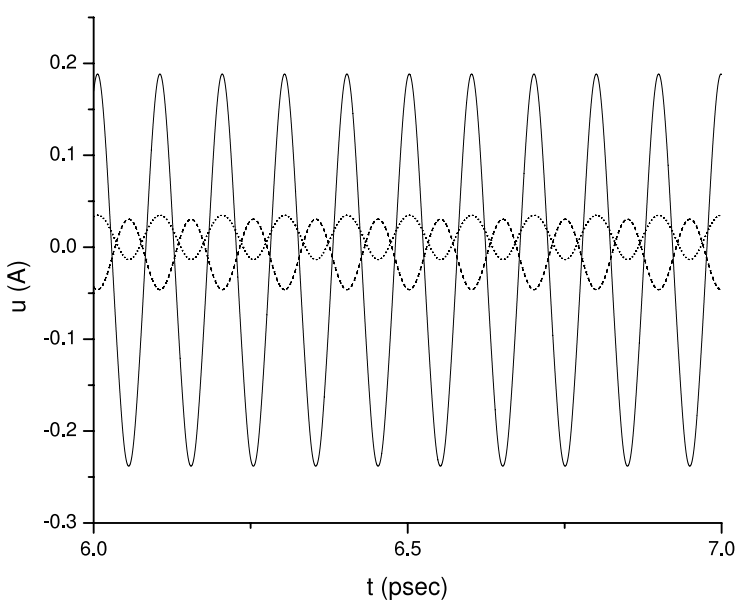

Fig. 5. DB in Ge: time dependence of vibrations of central atom (solid line) and the first non-central neighbors: a) along (111) axis (dashed line), b) perpendicular to (111) axis (dotted line) with the frequency $10.07 \mathrm{THz}$.

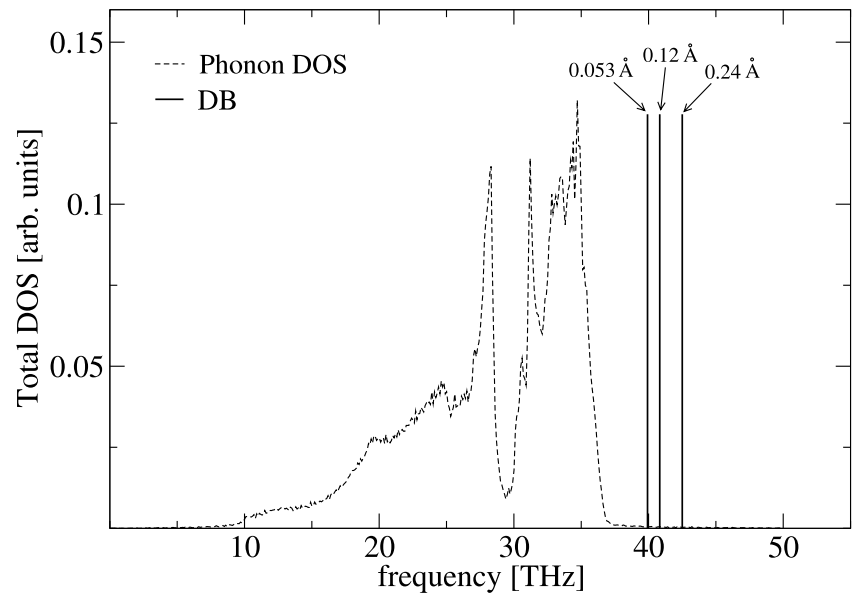

Fig. 6. Spectra of vibrations of carbon atoms in diamond with LCBOP interatomic potential for three DBs with different frequencies and amplitudes in $(1,1,1)$ direction. The vibrational amplitudes of central atoms of DBs indicated by arrows.

\section{Moving DBs with frequencies above phonon spectrum}

Up to now, immobile DBs have been discussed in this chapter. However, at least in fcc and in bcc lattices, the upward splitted DBs can move along the chains of the nearest neighbor atoms. It has been demonstrated via our $\mathrm{MD}$ simulations in $\mathrm{Cu}, \mathrm{Fe}$ and $\mathrm{Ni}$ (see Figures 7 - 9). In our simulations of mobile DBs in $\mathrm{Cu}$ the clusters with $60 \times 60 \times 41$ copper atom cells and $60 \times 60 \times 60$ iron atom cells were used; for an extended motion in iron, cluster $12 \times 12 \times 12$, prolonged in the (111) direction to have 200 atoms in the (111) chain, was used. To generate a moving DB we fix the initial atomic positions according to a standing $\mathrm{DB}$ and assign small initial velocities to two central atoms in the main atomic chain. The velocities of the generated mobile DB may be different but remain always small in comparison with the sound velocity. To calculate moving $\mathrm{DBs}$ in $\mathrm{Ni}$, a rectangular parallelepiped orientated and elongated along the moving direction (110) containing 40856 atoms was used. In this cluster the DB moves along the chain of 70 atoms; the center of the DB initially was at the position of the 20th atom of the chain

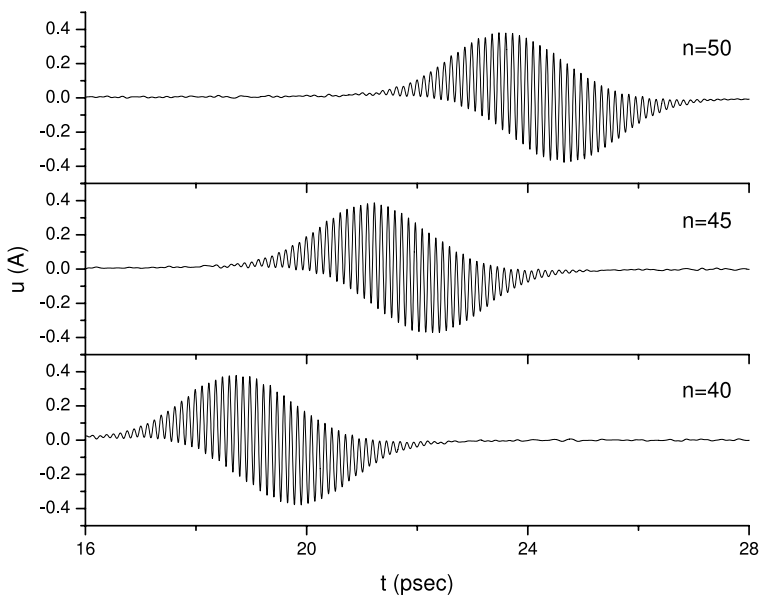

Fig. 7. Moving DB in $\mathrm{Cu}$ : time dependence of the vibrations of the atoms number 40, 45 and 50 in the (110) lattice chain (initially DB was localized close to the atom number $0(n=0)$ in the centre of the chain).

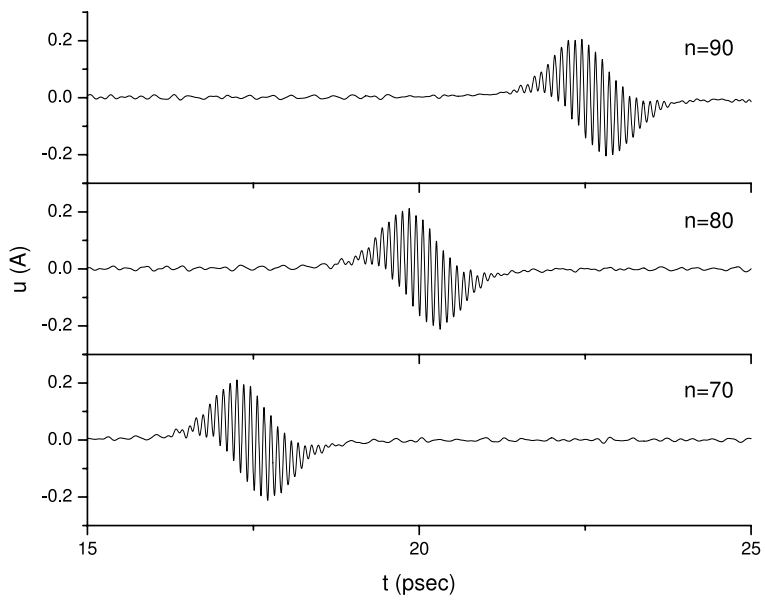

Fig. 8. Moving DB in Fe: time dependence of the vibrations of the atoms number 70, 80 and 90 in the (111) lattice chain (initially DB was localized close to the atom number $0(n=0)$ in the centre of the chain). 


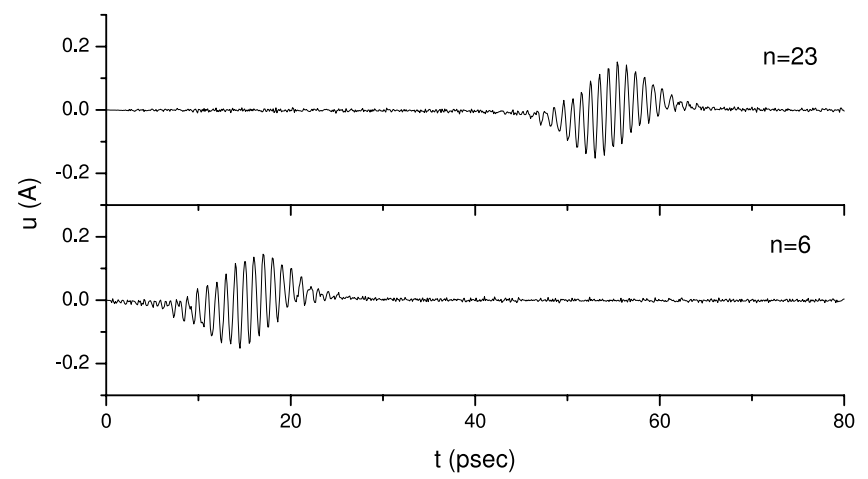

Fig. 9. Moving DB in Ni: the time dependence of the vibrations of the atoms number 6 and 23 in the (110) lattice chain (initially DB was localized close to atom number $0(n=0)-20$ th atom of the chain).

\section{Transverse discrete breathers}

There are systems in which the odd anharmonicities disappear due to symmetry arguments; at the same time, the quartic anharmonicity is non-zero and it is hard (positive). The examples of such systems are given by the linear atomic chains and by the planar atomic structures (e.g. graphene); in these systems odd anharmonicities disappear for vibrations in the transverse (out-of-chain and out-ofplane) direction. Therefore, one can expect that in these systems there can exist transverse anharmonic local modes with the frequencies above the maximum frequency of the corresponding phonons. The latter frequencies are usually smaller than the maximum frequency of longitudinal/ in-plane phonons. These modes fall in resonance with these phonons and can decay. However, unlike pseudolocal modes and like local modes the interaction causing the decay of these modes is anharmonic. We call them as the transverse discrete breathers (TDBs). The anharmonic interaction of TDBs with small vibrational amplitudes of atoms is weak. Hence, one can expect that the lifetime of such TDB may be rather long. Below we will consider TDBs in a monatomic chain and in graphene, both analytically and numerically. Our considerations confirm the aforesaid expectation.

\subsection{Transverse discrete breathers in anharmonic chain}

First we consider the anharmonic monatomic chain and examine the vibrations of its atoms in transverse $(y)$ directions. We suppose that the potential energy $U$ of the chain is given by the sum of pair potentials $V\left(R_{n, n^{\prime}}\right)$, where $R_{n, n^{\prime}}$ is the distance between the atoms $n$ and $n^{\prime}$. The latter potentials can be expanded into the series of atomic displacements. Denoting $d=\left(n-n^{\prime}\right) a, x=x_{n}-x_{n^{\prime}}, y=y_{n}-y_{n^{\prime}}$, where $a$ is the atom spacing, $x_{n}$ and $y_{n}$ are the longitudinal and transverse displacements of the atom number $n$ from its equilibrium position, we get $R \equiv R_{n, n^{\prime}}=\sqrt{(d+x)^{2}+y^{2}}$. As $y$ appear as $y^{2}$, any power expansion of $R$ will have only even powers of $y$. The same holds for $U$. This means that $U$ indeed has no odd anharmonic terms. This is a consequence of the symmetry of the chain with respect to the change of the sign of $y$.

Note one more property of the chain: the term in the expansion of $R$, quadratic with respect to $y$, has the same numerical factor as the term linear with respect to $x$. The same holds also for an arbitrary power of $R$. In the equilibrium state all linear terms with respect to the coordinates $x$ in the potential energy $U$ are cancelled. Therefore, all quadratic terms with respect to $y$ are also cancelled, i.e the frequencies of transverse vibrations in the pair potential approximation tend to zero. As a result the long-range fluctuations can be created with little energy cost and since they increase the entropy they are favored. This leads to the instability of the chain with respect to small transverse distortions (see in this connection the Mermin-Wagner theorem [39]).

To get the chain stable one needs to stretch it [6]. In this case the atom spacing $a$ is replaced by $a+s$, where $s$ is stretching. Then the terms in $U$ linear with respect to $x$ and $y^{2}$ are not cancelled any more. Therefore, the elastic springs for transverse vibrations are also nonzero and positive. This results in the appearance of transverse phonons with finite, although small for small stretching maximum frequency $\omega_{t m}$. As these phonons do not have any cubic anharmonicity, but have nonzero positive quartic anharmonicity, low-frequency TDBs with the frequency above the spectrum of transverse phonons should exist here. These modes fell to resonance with longitudinal phonons and therefore can decay. However there are two reasons for this decay to be be very slow: 1) the interaction of TDBs with longitudinal phonons, causing the decay is anharmonic and is relatively week, 2) due to low frequency, TDBs get to resonance with long-wave longitudinal phonons which weakly interact with the TDBs.

Let us consider the TDB in a monatomic chain with the Morse pair potential

$$
V=D\left(1-e^{\alpha(a-r)}\right)^{2}
$$

Here $D$ is the energy of dissociation, $\alpha$ is the parameter. We are using dimensionless coordinates with the units corresponding to $a=1$ and the value $\alpha=4$ of the Morse pair potentials of atoms in monatomic metals. For this potential only nearest-neighbor interactions are essential and only these potentials will be taken into account here. We also take for the mass units the mass of the atoms of the chain $(M=1)$. The dissociation energy is chosen so that the unit frequency will correspond to the maximum frequency of longitudinal phonons. In this case the potential energy of the stretched lattice is the sum of the following pair potentials (up to a constant term):

$$
V=\left(1-e^{-4(r-1)}\right)^{2} / 128-x\left(1-e^{-4 s}\right) e^{-4 s} / 16,
$$

where

. The last term in Eq. (7) accounts for the effect of the stretching force of the chain in $x$ direction - it changes the equilibrium distance of the atoms from $r=1$ to $r=1+s$. Let us expand the potential into the series of $x$ and $y$ and take into account up to the second-order terms with respect to $x$ and forth-order terms with respect to $y$. We get (up to a constant)

$$
V(x, y) \approx \frac{v_{1}}{8} x^{2}+\frac{v_{2}}{8} s y^{2}+\frac{v_{3}}{8} x y^{2}+\frac{v_{4}}{32} y^{4},
$$

where $v_{i}$ are dependent on stretching $s$ parameters. In the small $s$ limit $v_{i} \approx 1$. If $s=0.05$ then $v_{1} \approx 0.522, v_{2} \approx 0.707$, $v_{3} \approx 0.463, v_{4} \approx 0.441$.

The pair potential of the longitudinal vibrations alone is given by the first term in the right-hand side of Eq. (8). Vibrational frequencies of corresponding phonons are 
given by Eq. (1) with $K_{2}=v_{1} / 4$. The maximum frequency of longitudinal phonons corresponds to $k=-\pi$ and equals $\omega_{l m}=\sqrt{v_{1}}$. The transverse vibrations alone are described by the pair potential

$$
V(0, y)=\frac{v_{2} s}{8} y^{2}+\frac{v_{4}}{32} y^{4} .
$$

In harmonic approximation $\left(v_{4}=0\right)$ the frequencies of corresponding phonons are given by Eq. (1) with $v_{2} / 4$ instead on $K_{2}$. The positive quartic anharmonicity in Eq. (9) leads to appearance of the anharmonic modes [31] (called here as TDBs) with the frequencies $\omega_{0}=\omega_{t m} \sqrt{1+\varepsilon^{2} / 4}$ above the maximum frequency of transverse phonons $\omega_{t m}=\sqrt{s v_{2}}$ and with the displacements

$$
y_{n}(t) \approx(-1)^{n} A_{0} \cosh ^{-1}(\varepsilon n) \cos \left(\omega_{0} t\right) .
$$

Here $A_{0}$ is the amplitude of the central atom,

$$
\varepsilon=\sqrt{3 v_{4}} A_{0} / \omega_{t m}
$$
$\varepsilon=\sqrt{3 v_{4} A_{0} / \omega_{t m}}$
is the reversed size of the TDB (we use the discrete analog of the derived in Ref. [31] equation (47) for the difference in the displacements of two neighbouring atoms $\chi(x)$ ). These modes interact with longitudinal phonons and, therefore, they decay. To describe this decay we consider the longitudinal vibrations of atoms in the presence of the TDB. Taking into account Eq. (10) we replace $y$ by $y(t)$ in Eq. (8) and get the following pair potential for this motion:

$$
V(x, y) \approx \frac{v_{1}}{8} x^{2}+\frac{v_{3}}{16} x y^{2}(0)+\frac{v_{3}}{8} x y^{2}(0) \cos \left(2 \omega_{0} t\right) .
$$

The first term in the right-hand side of this equation gives the potential energy of the longitudinal vibrations alone in harmonic approximation. The second and the third terms describe the anharmonic interaction of these and transverse vibrations. At that the second term stands for a small local compression, while the third term describes the force with the frequency $2 \omega_{0}$ periodically changing in time. For all longitudinal phonons, except those with the resonant frequency $\omega_{k}=2 \omega_{0}$, this force causes forced vibrations of atoms with the frequency $2 \omega_{0}$. The resonant term causes the increase of the energy of phonons in time. From energy conservation law it follows that this energy comes from the TDB, i.e. the TDB decays.

\subsection{Decay rate of transverse discrete breathers in chain}

To find the rate of decay of TDBs, we are considering the equation of motion of the longitudinal phonon with the coordinate

$$
x_{k}=N^{-1 / 2} \sum_{n} x_{n} \sin (k n),
$$

where $N \gg 1$ is the number of atoms in the chain, $k=2 \pi m / N$ is the wave number of the phonon, $m=0, \pm 1, \pm 2, \ldots, x_{n}$ is the displacement of the atom $n$ from its equilibrium position (we use the periodic boundary conditions). In the case of pair potential (12) this equation reads

$$
\begin{array}{r}
\ddot{x}_{k} \cong-\omega_{k}^{2} x_{k}+\frac{V_{3}}{16 N} \sum_{n=-\infty}^{\infty} \sin (k n)\left(\left(y_{0, n+1}-y_{0, n}\right)^{2}-\right. \\
\left.-\left(y_{0, n}-y_{0, n-1}\right)^{2}\right) \cos \left(2 \omega_{0} t\right),
\end{array}
$$

where $\omega_{k}$ is the frequency of the longitudinal phonon $k$ given by Eq. (1) with $K_{2}=v_{1} / 4, y_{0, n} \equiv y_{n}(0)$ is the initial amplitude of the transverse $\mathrm{DB}$ on atom $n$ given by Eq. (10). The first term in the right-hand side of Eq. (14) stands for the harmonic force stemming from the first term in the right-hand side of Eq. (12) and the second term accounts for the anharmonic force stemming from the third term in the right-hand side of this equation (a small compression of the chain stemming from the second term in the right-hand side of Eq. (12) is neglected). The transverse amplitude $\left|y_{0, n}\right|$ slowly changes with $n$. Therefore, $y_{0, n+1}-y_{0, n} \cong 2 y_{0, n}$ and

$$
\left(y_{0, n+1}-y_{0, n}\right)^{2}-\left(y_{0, n}-y_{0, n-1}\right)^{2} \approx 4 \partial y_{0, n}^{2} / \partial n .
$$

Inserting Eq. (15) into Eq. (14) and taking into account Eq. (10), we get

where

$$
\ddot{x}_{k}+\omega_{k}^{2} x_{k} \cong C_{k} N^{-1 / 2} \cos \left(2 \omega_{0} t\right),
$$

$$
\begin{aligned}
C_{k} & \cong \frac{v_{3}}{4} A_{0}^{2} \sum_{n=-\infty}^{\infty} \sin (k n) \frac{\partial}{\partial n}\left(\frac{1}{\cosh ^{2}(\varepsilon n)}\right) \cong \\
& \cong-\varepsilon v_{3} A_{0}^{2} \sum_{n=0}^{\infty} \sin (k n) \frac{\sinh (\varepsilon n)}{\cosh ^{3}(\varepsilon n)} .
\end{aligned}
$$

By using the Green's function of harmonic oscillator $G_{k}(t)=\omega_{k}^{-1} \sin \left(\omega_{k} t\right)$, the solution of Eq. (16) can be presented in the following form:

where

$$
x_{k}(t)=x_{0, k} \cos \left(\omega_{k} t\right)+x_{k}^{\prime}(t)
$$

$$
x_{k}^{\prime}(t)=\frac{C_{k}}{\sqrt{N} \omega_{k}} \int_{0}^{t} \sin \left(\omega_{k}\left(t-t^{\prime}\right)\right) \cos \left(2 \omega_{0} t^{\prime}\right) d t^{\prime} .
$$

The first term in Eq. $(18)\left(\propto x_{0, k}\right)$ stands for free oscillations, while the second term $\left(x_{k}^{\prime}(t)\right)$ describes the excitation of vibrations by the transverse mode. The energy of the excited vibrations is given by the sum of the terms $\omega_{k}^{2} x_{k}^{\prime 2}$ averaged over period and summed over all phonons. This gives

$$
\begin{array}{r}
E(t)=\frac{1}{N} \sum_{k} C_{k}^{2}\left\langle\int_{0}^{t} d t^{\prime} \sin \left(\omega_{k}\left(t-t^{\prime}\right)\right) \cos \left(2 \omega_{0} t^{\prime}\right) .\right. \\
\left.\cdot \int_{0}^{t} d t^{\prime \prime} \sin \left(\omega_{k}\left(t-t^{\prime \prime}\right)\right) \cos \left(2 \omega_{0} t^{\prime \prime}\right)\right\rangle
\end{array}
$$

Let us take into account the relations:

$$
\begin{aligned}
& \sin \left(\omega_{k}\left(t-t^{\prime}\right)\right) \sin \left(\omega_{k}\left(t-t^{\prime \prime}\right)\right)= \\
& \quad=\left(\cos \left(\omega_{k}\left(t^{\prime}-t^{\prime \prime}\right)\right)-\cos \left(\omega_{k}\left(2 t-t^{\prime}-t^{\prime \prime}\right)\right)\right) / 2, \\
& \cos \left(2 \omega_{0} t^{\prime}\right) \cos \left(2 \omega_{0} t^{\prime \prime}\right)= \\
& \quad=\left(\cos \left(2 \omega_{0}\left(t^{\prime}-t^{\prime \prime}\right)\right)+\cos \left(2 \omega_{0}\left(t^{\prime}+t^{\prime \prime}\right)\right)\right) / 2 .
\end{aligned}
$$

At large $t$ due to the summation over $k$ and integration over $t^{\prime}$ and $t^{\prime \prime}$ only the term with argument $\left(\omega_{k}-2 \omega_{0}\right)\left(t^{\prime}-t^{\prime \prime}\right)$ can give a remarkable contribution to the energy $E(t)$; all other terms average out. This gives

$$
E(t) \cong \frac{1}{8 N} \sum_{k} C_{k}^{2} \int_{0}^{t} d t^{t} \int_{0}^{t} d t^{\prime \prime} \cos \left(\left(\omega_{k}-2 \omega_{0}\right)\left(t^{\prime}-t^{\prime \prime}\right)\right) .
$$

For small $\left|t^{\prime}-t^{\prime \prime}\right|$ being essential here one can replace the integral $\int_{0}^{t} \int_{0}^{t} d t^{\prime} d t^{\prime \prime}=\int_{0}^{t} d t_{1} \int_{-t^{\prime}}^{t^{\prime}} d \tau$ by the integral $\int_{0}^{t} d t_{1} \int_{-\infty}^{\infty} d \tau$, where $t_{1}=\left(t^{\prime}+t^{\prime \prime}\right) / 2, \tau=t^{\prime}-t^{\prime \prime}$. As a result, in the $t \rightarrow \infty$ limit the rate of increasing of the energy of longitudinal phonons, followed by a subsequent decrease of the energy of the TDB under consideration, equals

$$
d E(t) / d t=(\pi / 4 N) \Sigma_{k} C_{k}^{2} \delta\left(\omega_{k}-2 \omega_{0}\right) .
$$

Replacing here the sum by the integral and taking into account the relations $d m / d k=N / 2 \pi$ and $d k / d \omega_{k}=4 \omega_{k} / \sin k$ 
one finds

$$
d E / d t \approx\left(\omega_{0} v_{3}^{2} A_{0}^{4} / \sin k_{0}\right) \Phi^{2}(\Omega),
$$

where $\omega_{0}=\omega_{t m} \sqrt{1+\varepsilon^{2} / 4}, k_{0}=\arccos \left(1-8 \omega_{0}^{2} / v_{1}\right), \Omega=k_{0} / \varepsilon$, $\varepsilon=A_{0} \sqrt{3 v_{4}} / \omega_{t m}$

$$
\Phi(\Omega)=\int_{0}^{\infty} \frac{\sin (\Omega x) \sinh (x)}{\cosh ^{3}(x)} d x .
$$

The decay constant $\Gamma$ of the TDB is determined by the equation $\Gamma=E^{-1} d E / d t$. The energy of the TILM equals $E=\omega_{0}^{2} \sum_{n} y_{n}^{2}(0) / 2=\omega_{0}^{2} A_{0}^{2} / \varepsilon$. If to use the dissociation energy $D$ in electron-volts then we get

$$
\Gamma=\frac{\varepsilon v_{3}^{2} A_{0}^{2}}{\omega_{0} \sin k_{0}} \Phi^{2}(\Omega) .
$$

$\Gamma$ decreases with decreasing of the amplitude of the central atom $A_{0}$ of the TDB. To get an estimation we consider the TDB with the amplitude $A_{0}=0.03$ in the $5 \%$ stretched chain $(s=0.05)$. In this chain $\omega_{t m} \approx 0.1880, \varepsilon \approx 0.183, \omega_{0} \approx 0.1888$, which gives $\Omega \approx 6$ and $\Phi^{2}(\Omega) \approx 2 \cdot 10^{-5}$. As a result we get $\Gamma \approx 5 \cdot 10^{-9}$. This corresponds to a very long lifetime.

\subsection{Numerical study of transverse discrete breathes in the chain}

In our numerical study of transverse DBs we considered a 5\% stretched chain with 40000 atoms and with fixed ends. The small compression of the entire chain due to TDB was taken into account. The potential energy was described by the interactions of the nearest neighbors with the Morse pair potential given by Eq. (6). We have found that TDBs indeed exist in this chain and they have a long lifetime (see Figures $10-12$; one period of the TDB with the frequency $\omega_{0} \approx 0.1888$ corresponds to 33 time units). E.g. in our numerical simulations we have found that the TDB with $A_{0}=0.03$ and $s=0.05$ has the frequency $\omega_{0} \approx 0.1883$ and the reversed size parameter $\varepsilon \approx 0.113$. It decays very slowly: its amplitude diminishes less than $2 \cdot 10^{-8}$ for 1000 periods of vibrations. This corresponds to $\Gamma<2 \cdot 10^{-11}$, which indeed gives very long lifetime, in agreement with the conclusion of the theory.

The TDBs with the amplitudes $A_{0} \geq 0.05$ decay faster; the rate of their decay rapidly increases with the increasing of the amplitude. This is also in agreement with the theory.

Concerning the difference of the above-presented numerically-found values of the parameters $\varepsilon$ and $\omega_{0}$ from their theoretical values, we have found that these differences come from the neglecting of the local compression of the chain in the theory. This follows from the numerical simulations of TDBs in the chain rigid in $x$ direction (with $x$ coordinates of atoms being fixed at their values without TDB), performed by us. We have found the TDB in this chain with $\varepsilon \approx 0.183$ and $\omega_{0} \approx 0.1888$ in full agreement with the above theoretically found values of these parameters. This TDB does not decay, as it should according to the theory presented above. An account of the compression mentioned in the theory, allowing one to explain the values $\varepsilon \approx 0.113$ and $\omega_{0} \approx 0.1883$ found in simulations, is given in [21] in Appendix. Note that if to use these values of $\varepsilon$ and $\omega_{0}$ in Eq. (25) we get $\Gamma \approx 2 \cdot 10^{-13}$. This corresponds to an extremely long lifetime.

When describing TILMs, the dimensionless units were used. To give an idea about the actual values of energies of TILMs under discussion, we present here also the energy of a TILM in the dimensional units:

$$
E=32 D\left(A_{0} / a\right) \sqrt{S / 3 a}\left(4 s / a+3 A_{0}^{2} / a^{2}\right)
$$

E.g. for $D=10 \mathrm{eV}, A_{0} / a=0.05, s / a=0.05$ one gets $E=0.43 \mathrm{eV}$. A TILM with such energy may live more than one second supposing that $\omega_{l m} \sim 10^{13} \mathrm{sec}^{-1}$.

Above we described only standing TDBs. We also performed numerical study of transverse vibrations with nonzero total momentum in longitudinal direction and have found that TDBs can move along the chain (see Fig. 13). Depending on the value of the initial momentum, the velocity of TDBs can be smaller or larger. Only TDBs moving with small velocity as compared to the velocity of phonons were found.

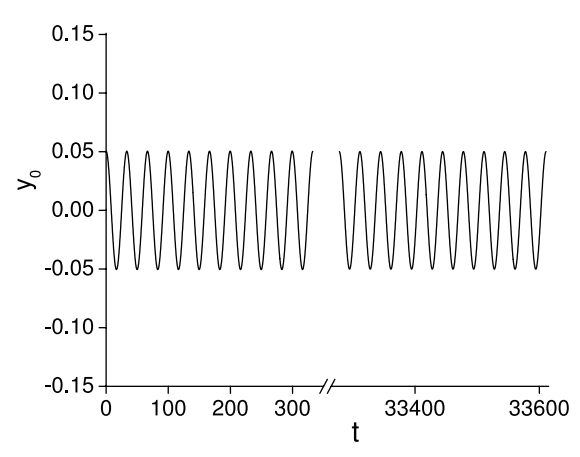

Fig. 10. Time dependence of the transverse shifts (in the units of atom spacing) of the central atom of a transverse DB in 5\% stretched monatomic chain; the initial shift of the central atom is 0.05 . First 10 periods and 10 periods after 1000 periods of vibration are presented.

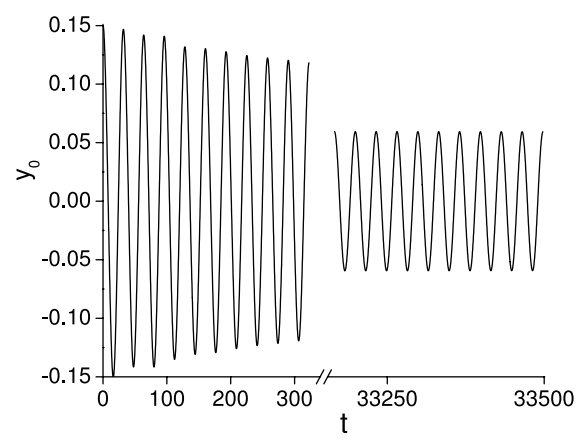

Fig. 11. The same as in Figure 10, but the initial shift of the central atom of a transverse DB is 0.15 .

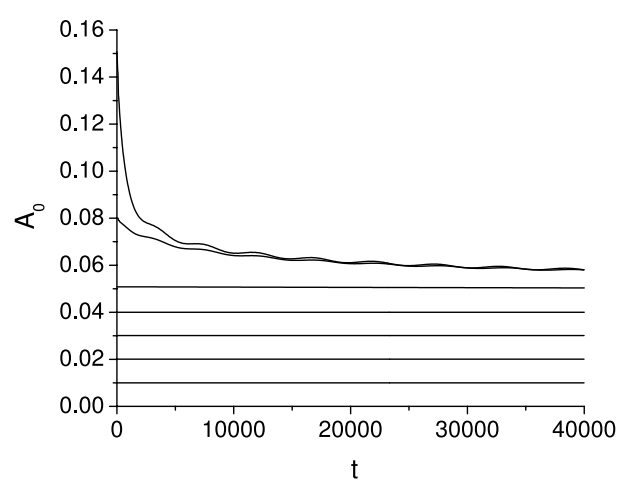

Fig. 12. Time dependence of the amplitudes of the central atom of transverse DBs in 5\% stretched monatomic chain for different initial shifts of the central atom. 


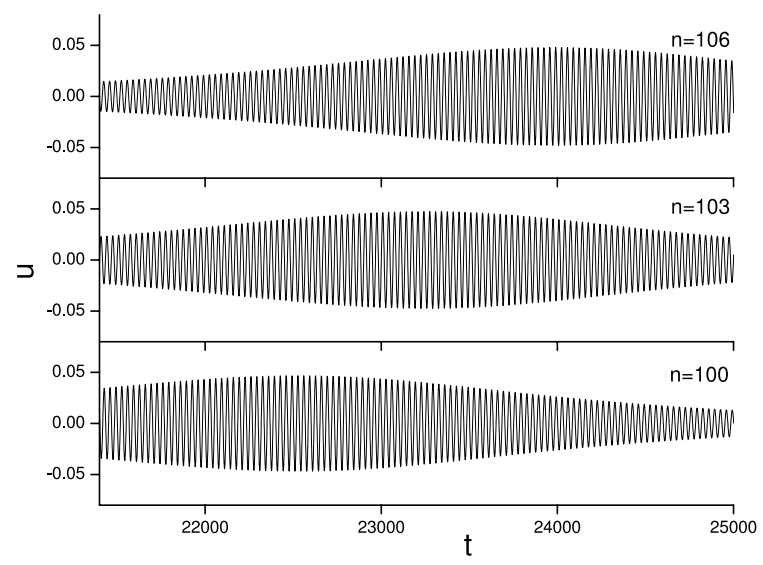

Fig. 13. Moving TDB in the 5\% stretched monatomic chain: time dependence of transverse shifts of the atoms number 100,103 and 106. Vibrations of the atom number 103 get maximum later than vibrations of the atom number 100 and earlier than vibrations of the atom number 106 (initially TDB was localized close to the atom number $0(n=0)$ in the centre of the chain).

\section{Graphene: out-of-plane vibrations}

The arguments presented in the beginning of Section 5 can readily be extended to planar atomic lattices. To be specific, we are considering here a graphene sheet. It consists of carbon atoms constituting the honeycomb-type periodical structure. Every carbon atom in this structure is connected with three neighboring atoms by chemical bonds formed by the $\mathrm{sp}_{2}$ hybrid orbitales.

We suppose that in equilibrium positions the graphene is situated in the $(x, y)$ plane. The distances between an atom and three neighboring atoms equal

$$
r_{n}=\sqrt{\left(b_{n} a+x_{n}\right)^{2}+\left(c_{n} a+y_{n}\right)^{2}+z_{n}^{2}}
$$

$(n=1,2,3)$, where $x_{n}, y_{n}$ and $z_{n}$ are the $x$-, $y$ - and $z$-components of the displacements of three neighboring atoms with respect to the central atom, $b_{1}=0, b_{2}=\sqrt{3} / 2, b_{3}=-\sqrt{ } 3 / 2, c_{1}=-1$, $c_{2}=1 / 2, c_{3}=1 / 2$. In the pair potential approximation the potential energy of the vibrations of atoms depends on the distances $R_{n}=r_{n}-$ a (explicit form of this energy in harmonic approximation, see e.g. in [1]). The expansion of these distances over the displacements $x_{n}, y_{n}$ and $z_{n}$ depends on the powers $R^{m}=\left(b x+c y+\left(x^{2}+y^{2}+z^{2}\right) / a\right)^{m}$ (here the subscript $n$ is omitted for simplicity). In the equilibrium position the $\propto R$ terms are cancelled. Therefore, the expansion of a pair potential over $z$ at $x=y=0$ starts with the positive quartic term $\propto z^{4}$, i.e. the $\propto z^{2}$ terms are absent. Consequently, in the pair potential approximation the $2 \mathrm{D}$ lattice is unstable with respect to small out-of-plane distortions. However, in graphene the atomic interactions are determined by covalent forces. These forces cause the stiffness of the planes with respect to the transverse short-range displacements of atoms [1]. In accordance with the Mermin-Wagner theorem [39] the plane of graphene remains unstable with respect to longrange transverse distortions, resulting in the appearance of ripples $[12,40]$. A stretching of the graphene sheet removes the ripples.

The elementary cell of graphene includes two atoms. Therefore, there are two branches of out-of-plane vibrations: acoustic and optic. One can expect the existence of two types of out-of-plane TDBs in graphene - with the frequency above the top frequency of acoustic out-of-plane phonons (the acoustic-like TDB) and with the frequency above the top frequency of optic out-of-plane phonons (the optic-like TDB). The acoustic-like out-of-plane TDBs can decay due to a relatively weak anharmonic interaction with the in-plane phonons and also due to in general stronger harmonic resonant interaction with out-of-plane optic phonons. However, the optic-like out-of-plane TDBs can decay only due to a relatively weak anharmonic interaction with in-plane phonons. Therefore, the optic-type out-of-plane TDBs should have a longer lifetime.

This conclusion is in agreement with our numerical modelling of out-of-plane TDBs in weakly-stretched (1.5 $\%)$ graphene. We have used the AIREBO potential and taken into account the cluster $246 \times 210 \AA(100 \times 50$ periods, 20000 atoms) with periodical boundary conditions. Initially we displaced out-of-plane six atoms of $\mathrm{C}_{6}$ ring according to the optical mode. We found that out-of-plane optic TDBs indeed exist; see Figure 14 (where the time-dependences of the displacements of one of the three equivalent atoms of the central $\mathrm{C}_{6}$ ring are presented; the other three atoms in this ring vibrate out of phase with the mentioned atom) and Figure 15 (where in-plane and out-of-plane phonons DOS and the spectrum of out-of-plane TDB for different initial amplitudes of carbon atoms are given).

Note that in strongly-stretched graphene there can exist also in-plane anharmonic localized vibrations (in-plane discrete breathers) [3, 27]. It appears (see Ref. [27]) that strong uniaxial stretching (along the zigzag or armchair direction) results in the opening of the gap in the middle of the phonon spectrum of graphene. This makes possible the existence of in-plane soft DBs (discrete breathers) with the frequency in the gap of the phonon spectrum. The authors $[3,27]$ have found that these modes remain relatively stable also when, due to a large stretching, their frequencies get into resonance with out-of-plane phonons.

Thus, the physical situation discussed in Refs. $[3,27]$ is reversed to the one considered here: the anharmonic in-plane modes with soft anharmonicity are in resonance with out-of-
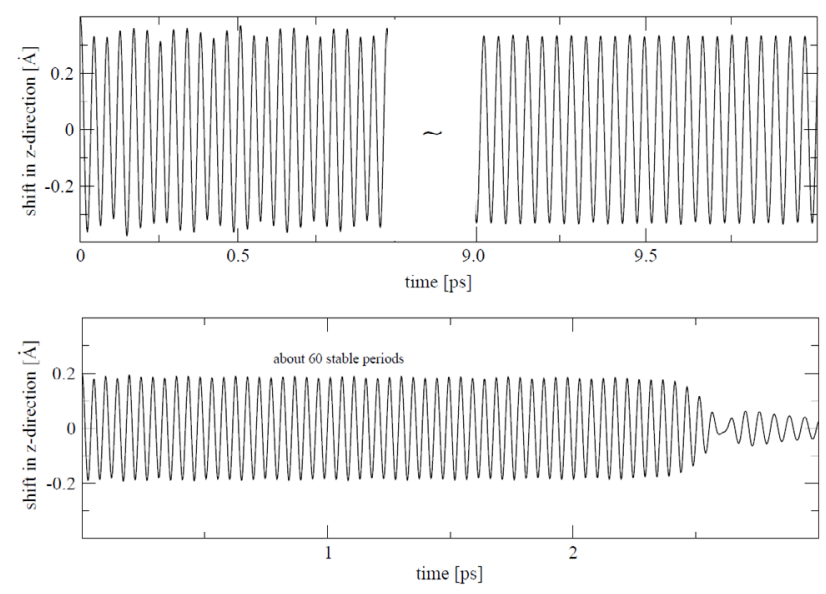

Fig. 14. Optical-type out-of-plane transverse DBs in graphene. The out-of-plane shifts (dependent on time) of one of three equivalent atoms of the central $\mathrm{C}_{6}$ ring is presented for initial amplitudes 0.4 $\AA$ (upper) and $0.2 \AA$ (bottom); the other three atoms in this ring vibrate out of phase with the atom mentioned. 
plane phonons, while out-of-plane TDBs considered here are in resonance with in-plane phonons. However, in both cases the lifetime of the modes is long.

\section{Concluding remarks}

In this communication we discussed discrete breathers in metals with frequencies above the top of the phonon spectrum. Two types of these excitation are recognized: 1) DBs appearing already at small energy, and 2) DBs existing only with large energy. The first type of DBs was found in metallic $\mathrm{Ni}$ and $\mathrm{Nb}$ [15]. The reason of existence of these excitations is the screening of the interaction between atoms by free electrons resulting in strong reduction of cubic anharmonicity near the equilibrium position of atoms. The second type of DBs was found in iron $(\mathrm{Fe})$ [20] and copper $(\mathrm{Cu})$ [19]. In these metals the superlinear response of the surrounding atoms on the local expansion of the lattice caused by the DB of a large amplitude leads to the essential reduction of this expansion and of the effect of odd anharmonicities. This in its turn results in the hardening of the effective elastic springs and in the increase of the frequency of an anharmonic modes with its amplitude.

We also have discussed DBs in semiconductors Ge and Si and in diamond, the frequencies of which too lie above the top of the phonon spectrum. The atomic forces in these crystals are essentially determined by the covalent chemical bonds resulting in strong orientation dependence. This dependence causes strong resistance of these systems with respect to the distortion of the main structural elements, p-tetrahedrons. Therefore the local expansion and softening caused by an anharmonic vibration in these crystals are essentially reduced already for small amplitudes.

We have shown that DBs in metals Fe [20], Cu and Ni [19] can move along the crystallographic directions corresponding to the chains of the nearest neighboring atoms. Especially impressive are moving $\mathrm{DBs}$ in $\mathrm{Cu}$ : they can transfer more than $5 \mathrm{eV}$ energy on large distances. The actual distance of propagation can be estimated as follows. According to [18, 22] the DBs can decay due to emission of pairs of phonons.

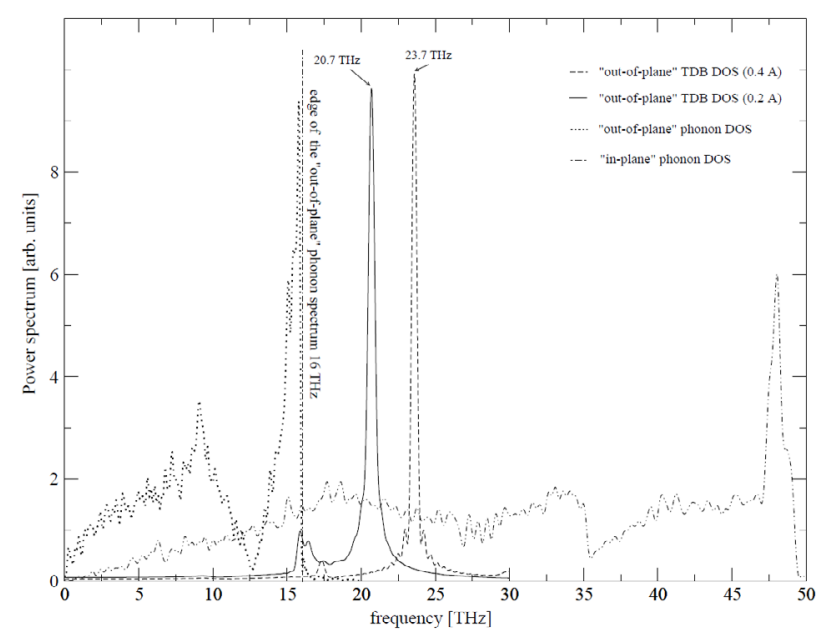

Fig. 15. In-plane and out-of-plane phonon DOS for the AIREBO potential and the spectrum of out-of-plane transverse DB for different initial amplitudes of carbon atoms.
At low temperatures the rate of emission should be threefour orders of magnitude less than the frequency of the DB [22]. Taking into account that energy of $\mathrm{DB}$ in $\mathrm{Cu}$ may many hundred times exceed the energy of a phonon one can expect that the life time of the decay may exceed $10^{-8} \mathrm{sec}$ and the distance of propagation may exceed $1 \mu$. This means that DBs can efficiently transfer large (as compared to typical phonon) energy on large distance.

We also have shown that due to symmetry arguments in linear monatomic chains and in planar monatomic structures (e.g. graphene) odd anharmonicities disappear for the motion of atoms normal with respect to the chain/ plane. However, even (quartic) anharmonicity exists and is hard (positive). As a result, the anharmonic localized modes of transverse (chain) or out-of-plane (graphene) vibrations, called transverse discrete breathers (TDBs) can exist in these systems with frequencies above the transverse/out-ofplane phonons. These vibrations have finite, although large lifetime due to slow anharmonic decay with creation of longitudinal phonons. For the case of a monatomic chain a theory is being developed which allows one to calculate the decay rate of TDBs. The theory predicts that the lifetime of the TDBs under consideration may be extremely long, longer than $10^{10}$ periods of vibration. The numerical modelling of these TDBs fully confirms this result. As we have shown these TDBs can move. The normal (out-of-plane) TDBs with the frequency above the phonon spectrum of out-of-plane phonons were also found for weakly-stretched graphene.

The symmetry argument presented here holds generally for linear atomic chains and planar monolayer atomic structures. Therefore, one can expect the existence of TDBs in any linear atomic chain and any planar monolayer atomic structure. Besides, one can expect that an analogous situation may exist in 3D lattices with dominating chain and layer structural elements, e.g. in graphite and in mica. In such lattices odd anharmonicities exist, but they may be essentially reduced for out-of-chain/plane vibrations.

In this connection we would like to point to investigations of M. Russell et al. of muscovite mica irradiated by high energy particles (see, e.g. [45]). The irradiation produces in mica very long black tracks parallel to the crystallographic planes and localized between the layers. These tracks are explained in Ref. [45] by creation in the recoil process of discrete breathers which propagate on long distance along the crystallographic directions. The layered structure on atomic scale plays a crucial role in the observed phenomena. Although it is not yet clear whether these long-living vibrational excitations are transverse or longitudinal, the necessity of the layered structure on atomic scale to observe the phenomenon gives support to an assumption that these excitation should contain remarkable transverse component.

Analogous TDBs may be expected to exist also for surface vibrations in crystals with planar atomic surfaces. The change of the potential energy of a surface atom for the shift in outer direction also does not have any odd terms with respect to the shift. This leads to the decrease of the entire oddanharmonicity effects for out-of-plane surface vibrations, which may result in the appearance of out-of-plane TDBs with frequencies above the maximum frequency of transverse surface phonons and with a long lifetime. 
It is known that next-to-next interactions in a chain can cause its zigzag-type secondary structure [49]. In such chains, e.g. in polyethylene, the mobile "solitons of tension" may exist [36] (involving the longitudinal-type motion of atoms). Besides, in such chains one should also expect the existence of transverse DBs. Here two types of transverse vibrations should be distinguished: parallel and perpendicular to the plane of zigzag. The odd anharmonic terms for the transverse displacements perpendicular to the plane mentioned are absent; however, for transverse displacements parallel to this plane these are present. If the zigzag angle is large, then the TDBs perpendicular to the plane of zigzag should exist. However, if this angle is small, then the odd anharmonicity for the displacements parallel to the plane of zigzag is reduced. In such chains the existence of both TDBs is expected. It is worthwhile to note that intrinsic localized modes (discrete breathers) are often used to explain the targeted energy transfer in such basic for living organism organic chains as DNA $[5,35]$ and proteins [44].

Acknowledgments. The research was supported by Estonian research projects SF0180013s07, IUT2-27 and by the European Union through the European Regional Development Fund (project 3.2.0101.11-0029).

\section{References}

1. V. Adamyan and V. Zavalniuk, J. Phys.: Condens. Matter 23 (2011) 015402.

2. Archilla, J. F. R., Coelho, S. M. M., Auret, F. D., Dubinko, V. I., Hizhnyakov, V.: Long range annealing of defects in germanium by low energy plasma ions. Physica D 297 (2015) 56-61.

3. J.A. Baimova, S. V. Dmitriev, K. Zhou, EPL 100 (2012) 36005.

4. Bickham, S. R., Sievers, A. J., Takeno, S.: Numerical measurements of the shape and dispersion relation for moving one-dimensional anharmonic localized modes. Phys. Rev. B 45 (18), 10,344 - 10,347 (1992)

5. M. Peyrard and A.R. Bishop, Phys. Rev. Lett., 62 (1989) 2755.

6. S. Cadet, Phys. Lett. A 121 (1987) 77.

7. Campbell, D. K., Flach, S., Kivshar, Y. S.: Localizing energy through nonlinearity and discreteness. Physics Today 57 (1), 43 - 49 (2004)

8. Chamati, H., Papanicolaou, N. I., Mishin, Y., Papaconstantopoulos, D. A.: Embedded-atom potential for Fe and its application to self-diffusion on Fe (100). Surf. Sci. 600 (9), 1793 - 1803 (2006)

9. Daw, M. S., Baskes, M. I.: Semiempirical, Quantum Mechanical Calculation of Hydrogen Embrittlement in Metals. Phys. Rev. Lett. 50 (17), 1285 - 1288 (1983)

10. Daw, M. S., Baskes, M. I.: Embedded-atom method: Derivation and application to impurities, surfaces, and other defects in metals. Phys. Rev. B 29 (12), 6443-6453 (1984)

11. Dolgov, A. S.: The localization of vibrations in a nonlinear crystal structure. Sov. Phys. Solid State 28, 907 - 910 (1986)

12. A. Fasolino, J.H. Los and M.I. Katsenelson, Nature Materials 6 (2007) 858.
13. Flach, S., Gorbach, A.: Discrete breathers - Advances in theory and applications. Phys. Rep. 467 (1-3), 1-116 (2008)

14. Flach, S., Willis, C. R.: Discrete breathers. Phys. Rep. 295 (5), $181-264$ (1998)

15. Haas, M., Hizhnyakov, V., Shelkan, A., Klopov, M., Sievers, A. J.: Prediction of high-frequency intrinsic localized modes in Ni and Nb. Phys. Rev. B 84, 144,303 (1-8) (2011)

16. Henry, B. R.: Local modes and their application to the analysis of polyatomic overtone spectra. J. Phys. Chem. 80 (20), 2160 - 2164 (1976)

17. Henry, B. R., Kjaergaard, H. G.: Local modes. Can. J. Chem. 80 (12), 1635 - 1642 (2002)

18. Hizhnyakov, V.: Relaxation jumps of strong vibration. Phys. Rev. B 53, 13,981 - 13,984 (1996)

19. V.V. Hizhnyakov, M. Haas, A. Shelkan and M. Klopov, in: J.F. R. Archilla, N. Jiménez, V.J. Sánchez-Morcillo, L. M. Garca-Raffi (Eds.), Quodons in mica: nonlinear localized travelling excitations in crystals, Springer Series in Material Science, 221 (2015) 229.

20. Hizhnyakov, V., Haas, M., Shelkan, A., Klopov, M.: Theory and molecular dynamics simulations of intrinsic localized modes and defect formation in solids. Phys. Scr. 89, 044,003 (1-5) (2014)

21. Hizhnyakov, V., Klopov, M., Shelkan, A.: Transverse intrinsic localized modes in monatomic chain and in graphene. Phys. Lett. A 380 (9-10), 1075 - 1081 (2016)

22. Hizhnyakov, V., Nevedrov, D., Sievers, A. J.: Quantum properties of intrinsic localized modes. Physica B 316-317, 132 - 135 (2002)

23. Hizhnyakov, V., Shelkan, A., Klopov, M.: Self-consistent theory of intrinsic localized modes: Application to monatomic chain. Phys. Lett. A 357 (4-5), 393-396 (2006)

24. Hizhnyakov, V., Shelkan, A., Klopov, M., Kiselev, S. A., Sievers, A. J.: Linear local modes induced by intrinsic localized modes in a monatomic chain. Phys. Rev. B 73, 224,302 (1-6) (2006)

25. Hizhnyakov, V., Shelkan, A., Klopov, M., Sievers, A. J.: Localized vibrations in perfect anharmonic lattices: Trapping on phonons. J. Lumin. 128 (5-6), 995-997 (2008)

26. Khadeeva, L. Z., Dimitriev, S. V.: Discrete breathers in crystals with $\mathrm{NaCl}$ structure. Phys. Rev. B 81, 214,306 (1-8) (2010)

27. L.Z. Khadeeva, S. V. Dmitriev, Yu. S. Kivshar, JETP Lett. 94 (2011) 539.

28. Kiselev, S. A., Bickham, S. R., Sievers, A. J.: Anharmonic gap modes in a perfect 1 -d diatomic lattice for standard two-body nearest-neighbor potentials. Phys. Rev. B 48 (18), 13,508-13,511 (1993)

29. Kiselev, S. A., Rupasov, V. I.: Stationary vibrational modes of a polyatomic chain of particles interacting via an even order potential. Phys. Lett. A 148 (6-7), 355 - 358 (1990)

30. Kiselev, S. A., Sievers, A. J.: Generation of intrinsic vibrational gap modes in three-dimensional ionic crystals. Phys. Rev. B 55 (9), 5755 - 5758 (1997)

31. Kosevich, A. M., Kovalev, A. S.: Self-localization of vibrations in a one-dimensional anharmonic chain. Sov. Phys. JETP 40 (5), 891 - 896 (1974) 
32. Lai, R., Sievers, A. J.: Nonlinear nanoscale localization of magnetic excitations in atomic lattices. Phys. Rep. 314 (3), $147-236$ (1999)

33. Los, J. H., Fasolino, A.: Intrinsic long-range bond-order potential for carbon: Performance in Monte Carlo simulations of graphitization. Phys. Rev. B 68, 024,107 (1-14) (2003)

34. MacKay, R. S., Aubry, S.: Proof of existence of breathers for time-reversible or Hamiltonian networks of weakly coupled oscillators. Nonlinearity 7 (6), 1623 - 1644 (1994)

35. P. Maniadis, B.S. Alexandrov, A.R. Bishop, and K. O. Rasmussen, Phys. Rev. E, 33 (2011) 011904.

36. L. I. Manevitch and A. V. Savin, Phys. Rev. E, 55 (1997) 4713.

37. Maradudin, A. A.: Theoretical and Experimental Aspects of the Effects of Point Defects and Disorder of the Vibrations of Crystals. In: F. Seitz, D. Turnbull (eds.) Solid State Physics, vol. 18, 19. Academic Press, New York (1966)

38. Maradudin, A. A., Montroll, E. W., Weiss, G. S., Ipatova, I. P.: Theory of Lattice Dynamics in the Harmonic Approximation. In: H. Ehrenreich, F. Seitz, D. Turnbull (eds.) Solid State Physics, Suppl 3, 2nd edn. Academic Press, New York (1971)

39. N. D. Mermin, Phys. Rev. 176 (1968) 250.

40. J. C. Meyer, A. K. Geim, M. I. Katsnelson, K. S. Novoselov, T. J. Booth and S. Roth, Nature 446 (2007) 60.

41. Mishin, Y., Mehl, M. J., Papaconstantopoulos, D. A., Voter, A. F., Kress, J. D.: Structural stability and lattice defects in copper: Ab initio, tight-binding, and embedded-atom calculations. Phys. Rev. B 63, 224,106 (1-16) (2001)

42. Ovchinnikov, A. A., Erihjman, N. S.: On vibrational energy localization at high levels of excitation. Vibrational excitons. Sov. Phys. Usp. 25 (10), 738 - 755 (1982)

43. Page, J. B.: Asymptotic solutions for localized vibrational modes in strongly anharmonic periodic systems. Phys. Rev. B 41 (11), 7835 - 7838 (1990)
44. S. Luccioli, A. Imparato, S. Lepri, F. Piazza and A. Torcini, Phys. Biol., 8 (2011) 046008.

45. F.M. Russell and C. J. Eilbeck, Europhysics Letters, 78 (2007) 10004.

46. Sage, M. L., Jortner, J.: Bond modes. Adv. Chem. Phys. 47, 293 - 323 (1981)

47. Sandusky, K. W., Page, J. B., Schmidt, K. E.: Stability and motion of intrinsic localized modes in nonlinear periodic lattices. Phys. Rev. B 46 (10), 6161 - 6168 (1992)

48. Sato, M., Hubbard, B. E., Sievers, A. J.: Nonlinear energy localization and its manipulation in micromechanical oscillator arrays. Rev. Mod. Phys. 78, 137 - 157 (2006)

49. A. V. Savin, L.I. Manevich, P.L. Christiansen and A. V. Zolotaryuk, Phys.-Usp. 42 (1999) 245.

50. Shelkan, A., Hizhnyakov, V., Klopov., M.: Self-consistent potential of intrinsic localized modes: Application to diatomic chain. Phys. Rev. B 75, 134,304 (1-6) (2007)

51. Sievers, A. J., Page, J. B.: Unusual anharmonic local mode systems. In: G. K. Horton, A. A. Maradudin (eds.) Dynamical Properties of Solids: Phonon Physics The Cutting Edge, vol. VII, pp. 137-255. North Holland, Amsterdam (1995)

52. Sievers, A. J., Takeno, S.: Intrinsic Localized Modes in Anharmonic Crystals. Phys. Rev. Lett. 61 (8), 970-973 (1988)

53. Tersoff, J.: New empirical model for the structural properties of silicon. Phys. Rev. Lett. 56 (6), 632-635 (1986)

54. Tersoff, J.: Modeling solid-state chemistry: Interatomic potentials for multicomponent systems. Phys. Rev. B 39 (8), 5566 - 5568 (1989)

55. Voulgarakis, N. K., Hadjisavvas, S., Kelires, P. C., Tsironis, G. P.: Computational investigation of intrinsic localization in crystalline Si. Phys. Rev. B 69, 113,201 (1-4) (2004)

56. Interatomic Potentials Repository Project. http://www.ctcms.nist.gov/potentials 